\title{
Intermittent release of transients in the slow solar wind: 1. Remote sensing observations
}

Article

Published Version

Rouillard, A. P., Davies, J. A., Lavraud, B., Forsyth, R. J., Savani, N. P., Bewsher, D., Brown, D. S., Sheeley, N. R., Scott, C. J., Harrison, R. A., Howard, R. A., Vourlidas, A., Lockwood, M., Crothers, S. R. and Eyles, C. J. (2010) Intermittent release of transients in the slow solar wind: 1. Remote sensing observations. Journal of Geophysical Research, 115. A04103. ISSN 0148-0227 doi: https://doi.org/10.1029/2009JA014471 Available at https://centaur.reading.ac.uk/7208/

It is advisable to refer to the publisher's version if you intend to cite from the work. See Guidance on citing.

Published version at: http://dx.doi.org/10.1029/2009JA014471

To link to this article DOI: http://dx.doi.org/10.1029/2009JA014471

Publisher: American Geophysical Union

All outputs in CentAUR are protected by Intellectual Property Rights law, including copyright law. Copyright and IPR is retained by the creators or other copyright holders. Terms and conditions for use of this material are defined in the End User Agreement. 


\section{www.reading.ac.uk/centaur}

\section{CentAUR}

Central Archive at the University of Reading

Reading's research outputs online 


\title{
Intermittent release of transients in the slow solar wind:
}

\section{Remote sensing observations}

\author{
A. P. Rouillard,,${ }^{1,2,3,4}$ J. A. Davies, ${ }^{2}$ B. Lavraud, ${ }^{3,4}$ R. J. Forsyth, ${ }^{5}$ N. P. Savani, ${ }^{5}$ \\ D. Bewsher, ${ }^{2,6}$ D. S. Brown, ${ }^{6}$ N. R. Sheeley, ${ }^{7}$ C. J. Davis, ${ }^{2}$ R. A. Harrison, ${ }^{2}$ \\ R. A. Howard, ${ }^{7}$ A. Vourlidas, ${ }^{7}$ M. Lockwood, ${ }^{1,2}$ S. R. Crothers, ${ }^{2}$ and C. J. Eyles ${ }^{2,8}$
}

Received 19 May 2009; revised 9 September 2009; accepted 22 October 2009; published 10 April 2010.

[1] The Heliospheric Imager (HI) instruments on board the STEREO spacecraft are used to analyze the solar wind during August and September 2007. We show how HI can be used to image the streamer belt and, in particular, the variability of the slow solar wind which originates inside and in the vicinity of the streamer belt. Intermittent mass flows are observed in HI difference images, streaming out along the extension of helmet streamers. These flows can appear very differently in images: plasma distributed on twisted flux ropes, V-shaped structures, or "blobs." The variety of these transient features may highlight the richness of phenomena that could occur near helmet streamers: emergence of flux ropes, reconnection of magnetic field lines at the tip of helmet streamers, or disconnection of open magnetic field lines. The plasma released with these transient events forms part of the solar wind in the higher corona; HI observations show that these transients are frequently entrained by corotating interaction regions (CIRs), leading to the formation of larger, brighter plasma structures in HI images. This entrainment is used to estimate the trajectory of these plasma ejecta. In doing so, we demonstrate that successive transients can be entrained by the same CIR in the high corona if they emanate from the same corotating source. Some parts of the streamers are more effective sources of transients than others. Surprisingly, evidence is given for the outflow of a recurring twisted magnetic structure, suggesting that the emergence of flux ropes can be recurrent.

Citation: Rouillard, A. P., et al. (2010), Intermittent release of transients in the slow solar wind: 1. Remote sensing observations, J. Geophys. Res., 115, A04103, doi:10.1029/2009JA014471.

\section{Introduction}

[2] Coronal streamers, observed in the electron-scattered continuum of the K-corona, usually appear as "helmet" structures consisting of a bright dome-shaped region in the lower corona surrounded by a less bright envelope which extends nearly radially away from the Sun [Koutchmy, 1992; Wang et al., 2007]. The latter forms rays which were first photographed by Annie Maunder in great detail

\footnotetext{
${ }^{1}$ Space Environment Physics Group, School of Physics and Astronomy, Southampton University, Southampton, UK.

${ }^{2}$ Space Science and Technology Department, Rutherford Appleton Laboratory, Chilton, UK.

${ }^{3}$ Université de Toulouse, UPS, Centre d'Etude Spatiale des Rayonnements, Toulouse, France.

${ }^{4}$ UMR 5187, Centre National de la Recherche Scientifique, Toulouse, France.

${ }^{5}$ Blackett Laboratory, Imperial College London, London, UK.

${ }^{6}$ Institute of Mathematical and Physical Sciences, University of Wales, Aberystwyth, UK.

${ }^{7}$ Space Science Division, Naval Research Laboratory, Washington, D. C., USA.

${ }^{8}$ Grupo de Astronomia y Ciencias del Espacio, Universidad de Valencia, Valencia, Spain.

Copyright 2010 by the American Geophysical Union. 0148-0227/10/2009JA014471
}

during the 1898 solar eclipse in India. Subsequent eclipse images and the advent of coronagraphs have shown that these rays are visible out to distances of 12 to $30 R_{\odot}$. While the central dome-shaped region corresponds to dense plasma trapped on closed magnetic field lines rooted in the photosphere, the rays are related to dense plasma escaping along magnetic field lines which extend into the interplanetary medium [Pneuman and Kopp, 1971; Wang et al., 1998]. The plasma moving at subsonic speeds along the open magnetic field lines of the helmet streamers is gradually accelerated to supersonic speeds as it propagates higher up in the corona and eventually becomes part of the solar wind. The elements of the solar wind plasma emitted by the same source region are located along an Archemedian spiral in the Parker model of the solar wind [Parker, 1958]. The belt of slow solar wind usually associated with helmet streamers near the Sun therefore extends into the interplanetary medium in a large-scale Archemedian spiral of slow flow. Fast solar wind reaches its terminal speed at 5-10 solar radii (or 1 to $3^{\circ}$ elongation in the plane of the sky) according to Grall et al. [1996]. In contrast, the slow solar wind probably reaches its terminal speed near 30-50 solar radii (or 8 to $13^{\circ}$ elongation in the plane of the sky) [Sheeley et al., 1997; Breen et al., 2002]. The Sun Earth Connection Coronal and 
Heliospheric Investigation (SECCHI) instrument package on the pair of NASA STEREO spacecraft images the slow solar wind during the final stages of its acceleration.

[3] The quasi-static morphology of streamers is reasonably well understood, following many experimental observations [Koutchmy and Stellmacher, 1976; Schmahl et al., 1992] and much numerical modeling [Mikić and Linker, 1996; Wang et al., 1997; Mikić et al., 2000; Saez et al., 2000; Thernisien and Howard, 2006]. Streamers are usually constrained to low heliocentric latitudes at solar minimum [McComas et al., 1998] but broaden and can form at high latitudes during solar maximum [McComas et al., 2001; Balogh and Smith, 2001]. These long-term variations are also relatively well understood [Wang and Sheeley, 2003]. A streamer can change in mass by as much as $50 \%$ between successive solar limb passages but is usually present for several solar rotations, in particular at solar minimum [Poland, 1978]. On much shorter time scales, a day or less, the emergence of coronal mass ejections (CMEs) can alter the geometry and location of streamers substantially by opening or closing the magnetic lines which define them [Suess et al., 2004].

[4] Evidence of plasma release from helmet streamers was seen in photometer observations from the Helios spacecraft [Jackson, 1991]. The three rotating Helios photometers, which remotely sensed the variability of the K-corona, observed small, dense plasma parcels propagating outward along solar radials rooted at the tip of helmet streamers. More recent observations made by the coronagraphs on board the Solar and Heliospheric Observatory (SOHO) [Brueckner et al., 1995] demonstrated that "plasma blobs" are continually emitted from helmet streamers [Sheeley et al., 1997; Wang et al., 1998, 2000; van Aalst et al., 1999] showing that classic CME onsets may not be necessary for helmet streamers to be "active" in terms of transient mass flow on very short time scales. These plasma blobs originate 3 to $4 \mathrm{R}_{\odot}$ from Sun center often as radially elongated structures above the cusps of helmet streamers. They have been found to move outward, maintaining constant angular extent and increasing in length in rough accord with their speed, which typically doubles from $150 \mathrm{~km} \mathrm{~s}^{-1}$ near $5 \mathrm{R}_{\odot}$ to $300 \mathrm{~km} \mathrm{~s}^{-1}$ near $25 \mathrm{R}_{\odot}$ [Sheeley et al., 1997]. Numerical models simulating plasma pressure variations around and inside helmet streamers confirm that packets of magnetic field lines should be expelled from the tip of streamers [Dahlburg and Karpen, 1997; van Aalst et al., 1999]. The plasma blobs may be created as plasma from the streamer is swept up by these disconnected magnetic loops, often forming a $\mathrm{V}$-shaped structure in coronagraph images [e.g., Wang et al., 1998]. The dominance of the magnetic field pressure over the plasma pressure near the Sun (i.e., low plasma beta) should force the plasma located downstream of the inferred reconnection point to be swept out by the outflowing disconnected field lines. An alternative explanation to the occurrence of transient mass flows from streamers may be the emergence of small helical fields or CMEs which are only partially observed in coronagraphs (as observed, for instance, by Rouillard et al. [2009b]). The plasma blob generated in these processes move higher up in the corona at the typical speed of the solar wind emanating from the streamer belt (300-400 $\left.\mathrm{km} \mathrm{s}^{-1}\right)$.

[5] At the coronal base $\left(2-15 \mathrm{R}_{\odot}\right)$, the eastern edge of the steamer belt, as viewed on Carrington maps, is a boundary between slow and fast solar wind sources. When these different flows are radially aligned by solar rotation, corotating interaction regions (CIRs) form higher up in the corona. Large coronal holes tend to emit fast solar wind and their location controls the formation of CIRs. The evolution of CIRs is reasonably well understood following intensive analytical [Lee, 2000] and numerical work [Pizzo and Gosling, 1994]. The detection of corotating variable flows in images taken by the Heliospheric Imager (HI) on board the STEREO spacecraft has been related to the entrainment of streamer blobs by CIRs during their outward propagation [Sheeley et al., 2008a, 2008b; Rouillard et al., 2008, 2009a]. This interaction between transients and the background solar wind is here investigated in great detail by using images taken by HI (paper 1) and by using in situ measurements of these same transients (paper 2).

[6] After a description of the instruments used in this paper (section 2), we present STEREO observations of the variability of streamer flows (section 3 ) over two solar rotations. Finally, we analyze the radial propagation of these plasma ejecta (sections 4,5 , and 6). In the second paper we use the estimated trajectories to carry out a detailed analysis of the in situ signatures of the imaged small-scale transients.

\section{Instruments: Orbital Configuration of the Spacecraft}

[7] The near-identical pair of NASA STEREO spacecraft, launched on 26 October 2006, orbit the Sun in the ecliptic plane at a heliocentric distance close to 1 astronomical unit (AU) such that one (the STEREO-A spacecraft) leads the Earth in its orbit, and the other (STEREO-B) trails the Earth. The orbits are such that the angle of separation between each spacecraft and the Earth increases by $22.5^{\circ}$ per year [Kaiser et al., 2008]. Along with a comprehensive complement of in situ instrumentation, each spacecraft carries a suite of imagers: the Sun-Earth Connection Coronal and Heliospheric Investigation (SECCHI) package [Howard et al., 2008]. SECCHI consists of an extreme ultraviolet imager (EUVI), two coronagraphs (COR-1 and COR-2), and the Heliospheric Imager (HI). The HI instrument on each STEREO spacecraft comprises two wide-field, visible-light imagers, HI-1 and HI-2. The HI detectors are charge-coupled devices (CCDs) with $2048 \times 2048$ pixels (note that $1024 \times 1024$ pixel synoptic images are routinely downlinked), where each pixel has a size of $13.5 \times 13.5 \mu \mathrm{m}$. HI-1 and HI- 2 observe in white light with a band pass of $630-730 \mathrm{~nm}$ and $400-1000 \mathrm{~nm}$, respectively [Eyles et al., 2009; Harrison et al., 2008; Brown et al., 2009]. HI-1 has a $20^{\circ}$ square field of view (fov), centered at $14^{\circ}$ elongation. The $70^{\circ}$ by $70^{\circ}$ fov of the outermost HI-2 camera is centered at $53.7^{\circ}$ elongation. Note that the elongation of a target is defined as the angle between the observer-Sun vector and the observer-target vector.

[8] Coronal electrons are by no means uniformly distributed along the line of sight of each HI image pixel. The $\mathrm{K}$-coronal light detected by $\mathrm{HI}$ is sunlight which has been Thomson-scattered by coronal electrons. Thomson scattering forces geometrical constraints on the part of the solar wind, that HI can observe. The convolution of Thomson scattering effects with the radial distribution of solar wind electron density and incident solar electromagnetic radiation shows that the brightness recorded by $\mathrm{HI}$ is maximal for 


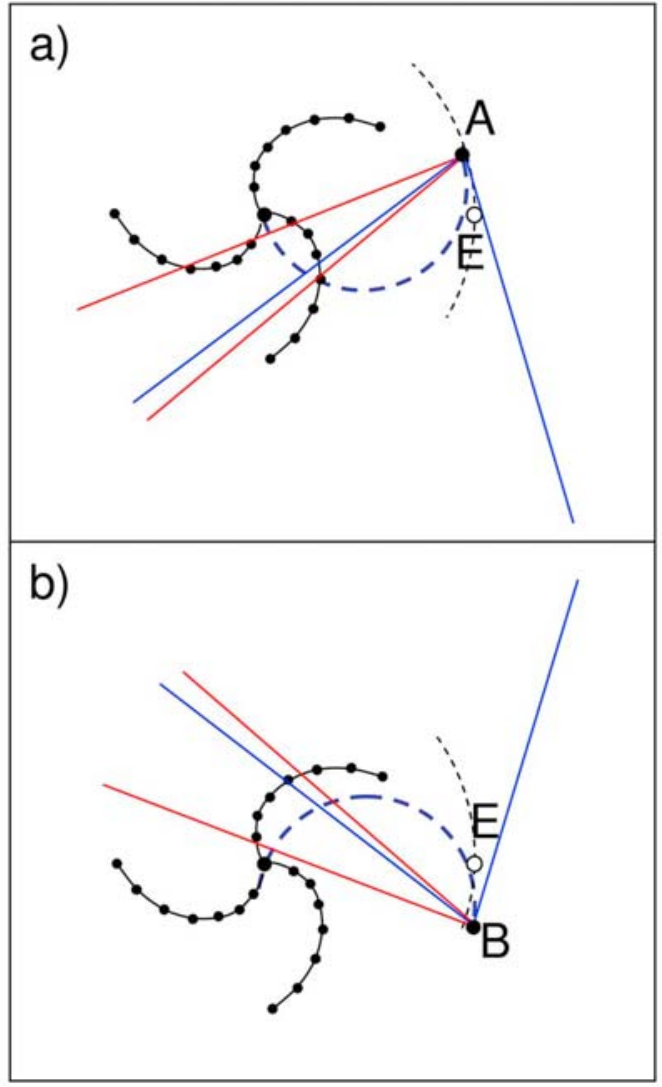

Figure 1. (a) A view of the ecliptic plane from the north showing the relative positions of the Sun (S), Earth (E), and STEREO-A spacecraft (A) in the solar ecliptic plane on 15 September 2007. (b) The same as Figure 1a but for STEREO-B (B). The angular extents of the fovs of the HI-1 and HI-2 cameras in the ecliptic plane are delimited by red and blue lines, respectively. The intersection of the Thomson sphere with the HI fov in that plane, for both the STEREO-A and -B spacecraft, is shown as a blue dashed semicircle (TS). The spiral configuration of plasma elements (small black dots) emitted by the same source regions is shown for a three sector structure.

electrons situated inside the volume of a sphere for which the Sun-spacecraft segment is the diameter; this sphere was termed the Thomson sphere (TS) by Vourlidas and Howard [2006]. Figure 1a presents a view of the ecliptic plane from above of the orbital configuration of the Sun (S), Earth (E) and STEREO-A (A) in the ecliptic plane on 15 September 2007. Figure $1 \mathrm{~b}$ is the same as Figure $1 \mathrm{a}$ but shows the location of the STEREO-B spacecraft (B). Figures $1 \mathrm{a}$ and $1 \mathrm{~b}$ also show the intersection of the Thomson sphere with the ecliptic which lies within the HI fov, for the appropriate STEREO spacecraft. Hence while it is hard to distinguish in the SOHO or STEREO coronagraph images whether a CME is propagating on the near or far side of the plane of the sky, HI will mostly be sensitive to electron density variations forced by transients directed at longitude angles less than $90^{\circ}$ relative to the imager. Vourlidas and Howard [2006] showed that the brightness of a $\mathrm{CME}$ remains relatively constant during its radial outward propagation inside this sphere; we refer the reader to their thorough discussion.
[9] The HI instruments, like coronagraphs, observe sunlight scattered off the F-corona as well as the K-corona, the former being light scattered by dust rather than solar wind electrons. The intensity of the K-corona signal measured by the HI instruments is small compared with that of the stars and the Fraunhofer (F)-corona. However, as the F-corona does not evolve significantly over time scales of the order of days, its contribution can be largely removed by either the use of the running difference technique or by subtracting the minimum value of the brightness measured in each pixel over an appropriate interval. Running difference images, obtained by subtracting the preceding image from the current one, are particularly useful as they reveal features that are propagating through the fov.

[10] Figure 2 presents a composite arrangement of background subtracted images of the $\mathrm{K}$-corona taken by the COR-1, COR-2 and HI-1 cameras of the SECCHI package on 23 September 2007. The corona is shown in a helioprojective cartesian latitude versus longitude plot. The images extend out to $\sim 24^{\circ}$ longitude off the eastern and western limbs of the Sun. Images off the eastern solar limb (left-hand side) are taken from the STEREO-A spacecraft and those off the western limb (right-hand side) are from STEREO-B; hence only half of each COR-1 and COR-2 image is used. The streamer rays emanating from the eastern limb of the Sun can be seen clearly out as far as $15^{\circ}$. If we assume that streamers are seen in detail inside the TS [Vourlidas and Howard, 2006]; a helioprojective longitude of $15^{\circ}$ corresponds to distances inside the TS of at least $\sim 54 \mathrm{R}_{\odot}$ away from the Sun. Hence HI-1 allows observations of streamer rays up to those distances where the slow solar wind has typically reached its terminal speed. In this paper we make extensive use of $\mathrm{HI}$ images to study streamer dynamics and the associated variable solar wind which has its source in these streamers.

\section{Transient Releases Inside the Streamer Belt}

[11] The region of slow solar wind associated with the coronal streamers (i.e., the streamer belt) can undergo large latitudinal excursions. These excursions are the result of nonaxisymmetric magnetic fields [Wang et al., 2006] developing at low solar latitudes during periods of weaker polar magnetic field [e.g., Sheeley, 2008]. The warping of the streamers and the Heliospheric Current Sheet (HCS), which is usually embedded in the streamer belt, leads to the formation of sector structures at solar equatorial latitudes. Two and four solar wind streams usually form during solar minimum. However in recent years in situ data recorded at Earth, as well as numerical models of the solar corona, point to the Earth often experiencing three corotating streams per solar rotation [Temmer et al., 2007]; as we shall see HI also observed this three-stream structure.

[12] The streamers will not appear when located outside the TS but as they enter the sphere their brightness is significantly enhanced. The electron density of the background solar wind is greatest on the TS (point of closest approach to the Sun) along the line of sight and the part of the streamer located on the sphere is consequently observed in great detail. We note that transients released at longitudes between the TS surface and the Imager, may become brighter than the part of the streamers located on the TS, depending on 


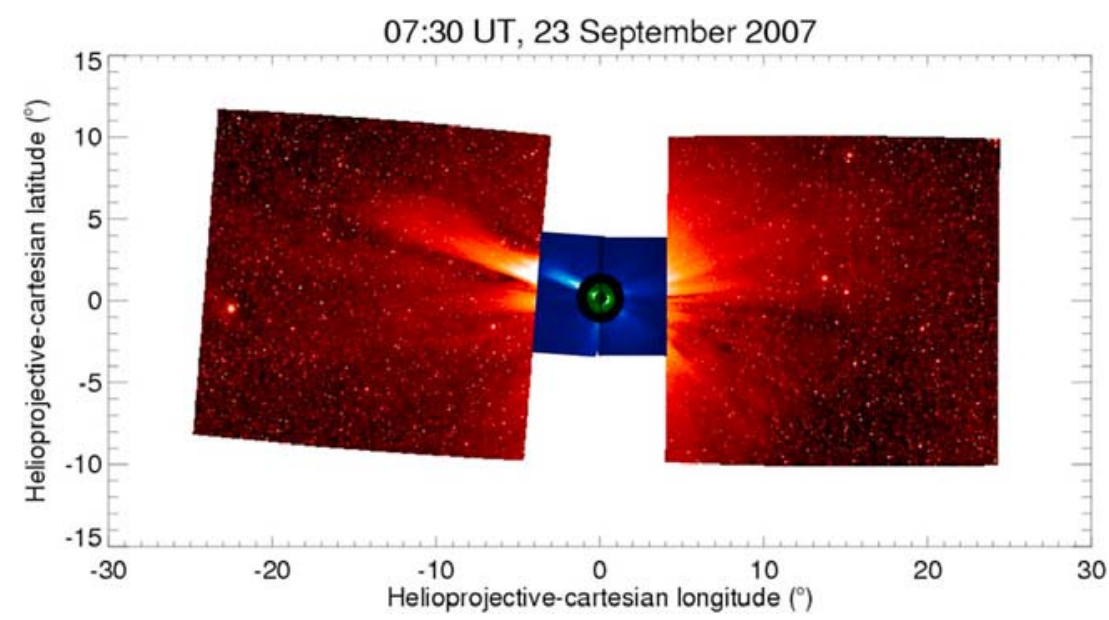

Figure 2. A composite of SECCHI images projected onto the plane of the sky. Images off the eastern solar limb (to the left of center) are from HI-1A (red) and COR-1/2A (green/blue) images; the K-corona off the western limb (to the right of center) is imaged by HI-1B and COR-1/2B. Note that only the relevant half of each COR image is shown.

their relative density along the line of sight. Assuming therefore that the part of streamers imaged by $\mathrm{HI}$ is located on the TS, a constant radial distance of $\sim 20 \mathrm{R}_{\odot}$ on the TS corresponds to an arc at near constant elongation of $5^{\circ}$. Figure 3 is a difference image obtained from two $40 \mathrm{~min}$ cadence HI-1A images taken at 0650 UT and 0730 UT on 23 September 2007. The two arcs plotted on this image bound a band of near constant $5^{\circ}$ elongation. This band can be plotted vertically with time in a synoptic map format. Such a map of coronal variability is presented in Figure 4a. The slow solar wind reaches its terminal speed at around $40 \mathrm{R}_{\odot}$; hence we are here extracting observations from a region half way through the acceleration phase. The distance

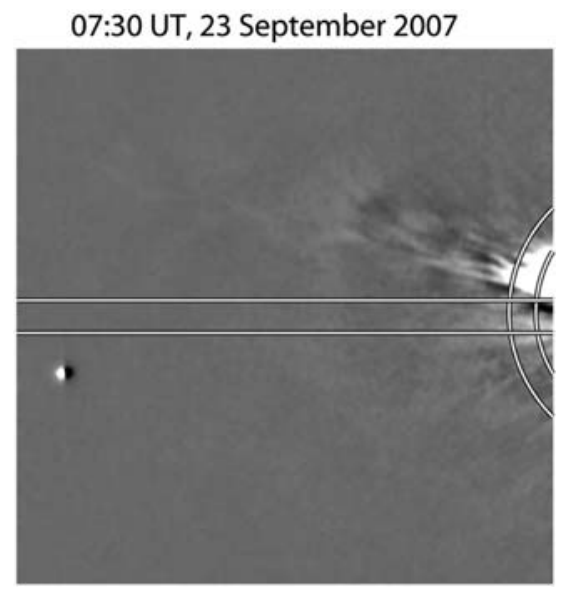

Figure 3. A running difference image obtained from HI-1A images recorded on 23 September 2007. The horizontal lines mark the limits of the band (portion) of difference image used to plot the vertical strip of data used at 0730 UT on 23 September 2007 in the J-map shown in Figure 5a. The two arcs mark the limits of the band of difference image used to create the vertical strip of data at 0730 UT on 23 September 2007 in the latitude versus time map shown in Figure 4a. of $20 \mathrm{R}_{\odot}$ was chosen because the brightness of streamers is still high at this short distance away from the Sun and fast solar wind has almost certainly not yet had time to catch up with the slow solar wind. Any variations in electron density originate therefore from varying solar wind properties at the coronal base; these variations can be caused by transients propagating along the streamer rays observed by HI (on the TS) or by transients propagating along longitudes situated between the TS surface and the Imager. Figure 4a presents 44 days of data covering parts of two consecutive Carrington Rotations (CR 2060 and CR 2061). In essence Figure 4a
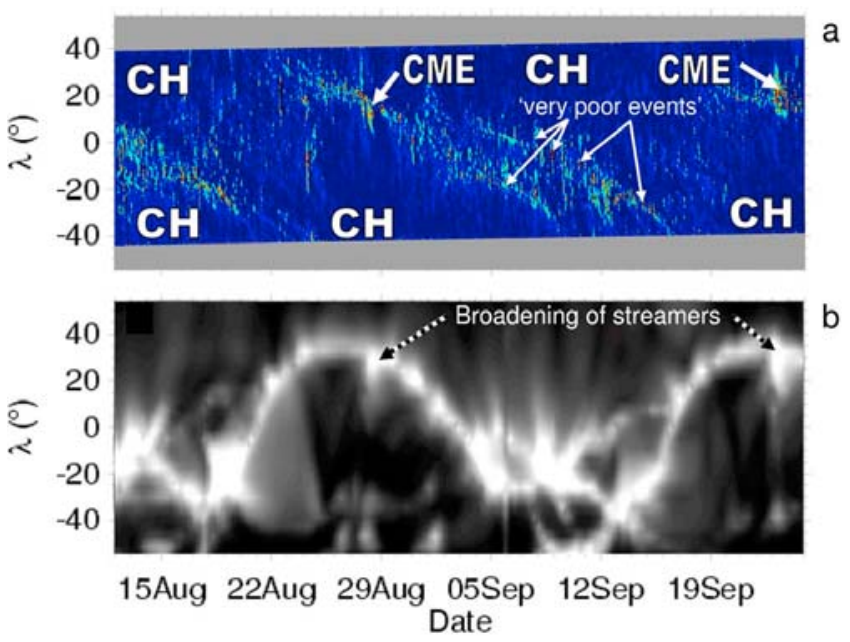

Figure 4. (a) The evolution of the streamer belt at $5^{\circ}$ elongation, as observed by HI-1 on STEREO-A, plotted in a latitude $\left(\lambda\left({ }^{\circ}\right)\right)$ versus time format. Uniform fast flows from coronal holes $(\mathrm{CH})$, recurring $\mathrm{CMEs}$, and "very poor events" or "blobs" can seen on this map. (b) Density predictions from 3-D MHD coronal modeling for that elongation off the eastern solar limb. The white areas correspond to dense solar wind, whereas the black area correspond to more tenuous wind. 
equates to a solar longitude versus latitude map, with orange/red areas marking the regions of most intense brightness increases (and thus electron density) between two consecutive images. The heliographic latitude is estimated by computing the intersection of the TS with a sphere centered at the Sun and of radius $20 \mathrm{R} \odot$.

[13] The region of greatest coronal intensity variations appears to occupy a belt; the heliographic latitude of this belt varies with time. The heliocentric latitude of the bright streamer on the eastern limb of the Sun in the HI-1A and COR-1/2A images in Figure 2 is the same as that of the belt of maximum coronal brightness variation inferred from Figure 4a on 23 September $\left(\lambda \sim 20^{\circ}\right)$. The thin belt of maximum brightness variability characterized by these orange/red areas in Figure 4a marks the latitudinal position of the densest part of streamer rays. Undisturbed flows can be seen on this map at high heliographic latitudes and are associated with coronal hole $(\mathrm{CH})$ flows.

[14] We can compare the latitudinal variation of the band of intense variability seen in Figure 4a to that of the location of the streamer belt predicted by three-dimensional magnetohydrodynamic (3-D MHD) modeling of the corona. The predictions of the solar wind speed and density from the Potential-Field Source Surface (PFSS) model of WangSheeley-Arge [Wang et al., 1990; Arge and Pizzo, 2000] are extended into the interplanetary medium using the ENLIL 3-D MHD numerical model of the solar wind [Odstrčil and Pizzo, 1999] Model predictions are available from the Community Coordinated Modelling Center. The solar wind density during CR 2060 and CR 2061 was simulated using the PFSS solar wind speed predictions based on smoothed and filtered magnetograms from the National Solar Observatory (NSO) at Kitt Peak (the preparation of these magnetograms was carried out by N. Arge). The PFSS model constructs a spherical harmonic representation, having interpolated the $\mathrm{NSO} 1^{\circ}$ resolution magnetograms onto a grid with $2.5^{\circ}$ resolution. The numerical code outputs the magnetic field vector, solar wind velocity vector, and plasma number density over the heliocentric distance range from 0.1 to $2.3 \mathrm{AU}$. This numerical model has been applied successfully to such topics as space weather forecasting, and has been used in the study of solar wind-magnetosphere coupling [Luhmann et al., 2004] and cosmic ray modulation [Rouillard and Lockwood, 2007].

[15] We present in Figure 4b the PFSS-ENLIL predictions of the solar wind density covering the same heliocentric latitude range and time interval as shown in Figure 4a. The latitudinal variation of the streamer belt as imaged by HI-1A is well replicated by the 3-D modeling. In particular, the large latitudinal excursions of the streamers observed between 19 and 23 August and one rotation later (i.e., 27 days later), between 15 and 20 September, are predicted by the model.

[16] Figures $4 \mathrm{a}$ and $4 \mathrm{~b}$ provide information on different time scales. Figure $4 \mathrm{a}$ is obtained from running difference images obtained at 40 min cadence and therefore only variations in the brightness of streamers over a $40 \mathrm{~min}$ interval will be recorded. Parts of the streamers which do not vary in brightness (time-dependent) or spatial position over this interval will be absent in Figure 4a, whereas Figure 4 b reveals the location of all parts of the streamer belt.

[17] Figure 4 shows that the thickness of the streamers along the HI line of sight is reduced when the belt undergoes large latitudinal excursions; thus the integrated brightness of the streamers is lower. Such periods are generally marked by lower intensities in Figure 4a (such as between 21 and 24 August 2007). Conversely, slower changes in the latitudinal location of the streamer belt will lead to more electrons contributing to the intensity along the line of sight, and the streamers will appear brighter. A bifurcation of the streamers is observed by HI-1A between 4 and 16 September 2007; this is confirmed by the PFSS-ENLIL model.

[18] Figure 4 provides information on the latitudinal width of the densest part of the streamer belt and in particular the latitudinal width of the largest of these white-light transients. We find that plasma parcels extend over a latitude width of $\sim 5-20^{\circ}$ which compares favorably with previous observations of intermittent coronal outflows [Sheeley et al., 1997; Wang et al., 2000]. During this early stage of the STEREO mission, the longitudinal separation was $17^{\circ}$ between STEREO and SoHO so that the planes of the sky of STEREO and SoHO coronagraphs were in direct proximity. We therefore decided to compare the occurrence of the sudden variations in coronal brightness observed by HI-1A with the SoHO LASCO CME catalogue created by Yashiro et al. [2004] (and held at http://cdaw.gsfc.nasa.gov/CME_list/). Seventy-seven listed transients emerge off the east limb of the Sun between $\mathrm{PA} \geq 45^{\circ}$ and $\mathrm{PA} \leq 135^{\circ}$ (the range of $\mathrm{PAs}$ covered by Figure $4 \mathrm{a}$ ) during the interval of time presented in Figure 4a. Seventy-eight percent of these transients are classified as "very poor events," have limited latitudinal extent $\left(<20^{\circ}\right)$, and are poorly resolved in LASCO images. Examples of "very poor events" listed by Yashiro et al. [2004] and identified as red areas on the map are shown in Figure 4a; they are located within the band of streamer belt variability. The longitude width of these transients may or may not extend over a similar angular range than their latitudinal width, we cannot say with single point observations from white-light integrated images, moreover the nature or origin of these streamer blobs is not well understood. Our interpretation that the transients observed in Figure $4 \mathrm{a}$ are propagating along the streamer rays results from the correspondence between the PA variation at which transients emerge and the PA variation of the streamer belt location; it is very likely that some of the transients observed in Figure 4a are propagating at longitudes well inside the sphere and emerged from other parts of the streamer belt.

[19] The large intensity variations observed by $\mathrm{HI}$ in the streamer belt reveal the variability and discontinuous nature of solar wind outflow. This is to be anticipated as the slow solar wind tends not only to be denser but also more variable. It is well known that in situ observations of the slow solar wind show that it is intrinsically more variable than the fast solar wind [Zurbuchen et al., 2002]. Hence the most intense variations observed in the difference images are likely to be related to the variability of the slow solar wind associated with the streamers. The densest solar wind is mainly observed near the center of the streamer belt inside the so-called "plasma sheet" where the Heliospheric Current Sheet (HCS) is usually located [Wang, 1994]. The thin belt of intense brightness variations seen in Figure $4 \mathrm{a}$ is likely to be mainly associated with the plasma sheet embedded in the streamer belt of Figure $4 \mathrm{~b}$. We associate the intermittency of streamer brightness variation with the streamer blobs observed in great detail in the LASCO coronagraphs on 


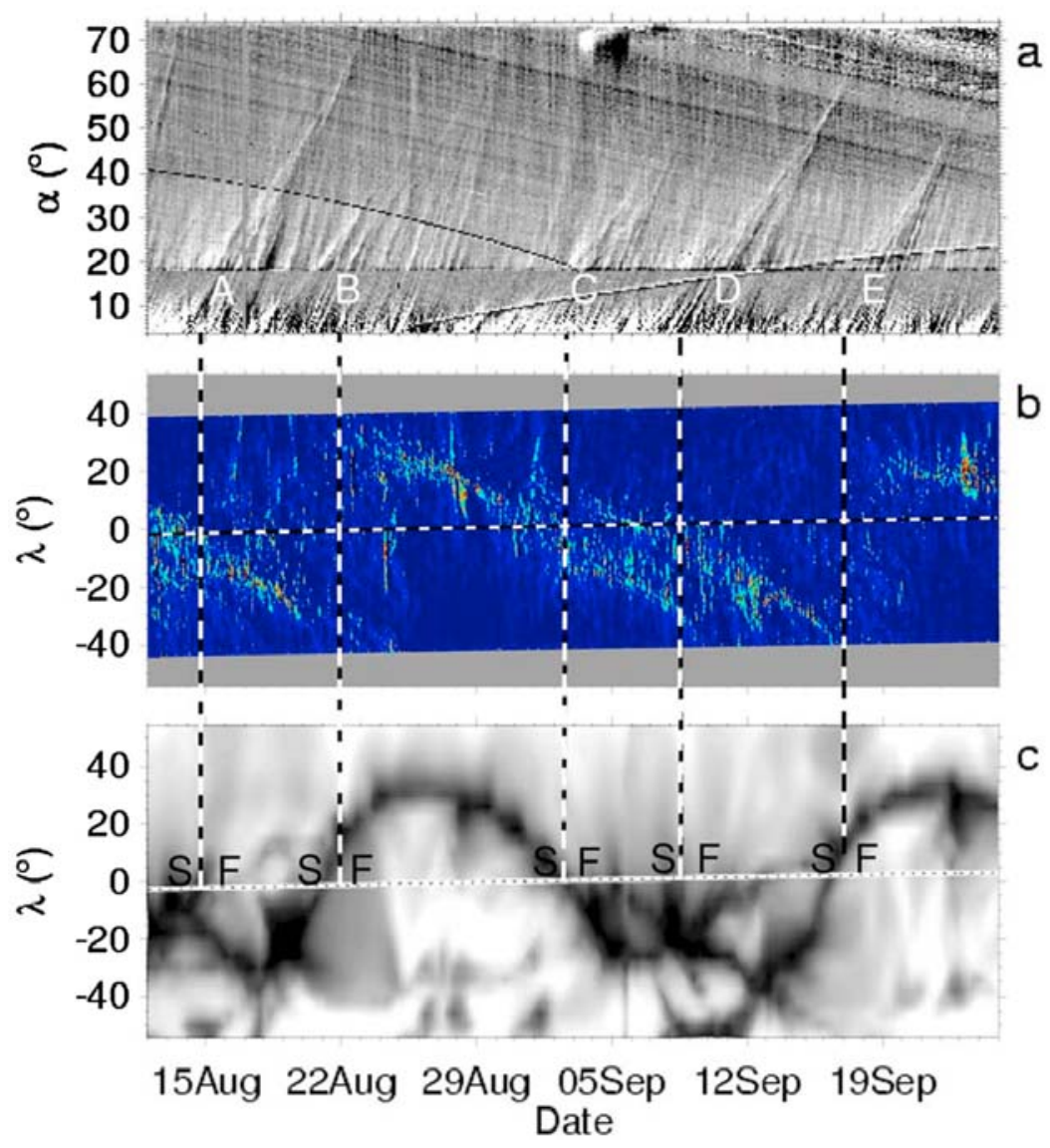

Figure 5. (a) Elongation ( $\alpha$, ranging from $4^{\circ}$ to $\left.74^{\circ}\right)$ versus time plot along the center of the HI fov on STEREO-A, derived from a sequence of HI-1/2A difference images. (b) The same as Figure 4a. (c) The radial speed predictions from the 3-D MHD coronal modeling. The latitudinal excursions of the streamers are shown as vertical dashed lines and mark the times of expected CIR formations. The varying latitude of the Earth is shown by a black-dotted white line in Figures $5 \mathrm{~b}$ and $5 \mathrm{c}$.

SOHO [Wang et al., 1998, 2000]. There are two larger red/ blue areas visible in Figure $4 \mathrm{a}$, emerging from the streamer belt on 28 August and 27 days later on 23 September at $20^{\circ}$ of latitude (marked as CME events in Figure 4a). These two features, manifested as widenings of the streamer belt by the 3-D MHD modeling, corresponded to intense signatures in the COR-1/COR-2 from the STEREO spacecraft and SOHO $\mathrm{C} 2$ and $\mathrm{C} 3$ coronagraph images. Two twisted structures reminiscent of flux ropes are visible in the COR-2 and LASCO/C3 images, which have been interpreted as "poor events" in the SOHO CME catalog, are probably east limb $\mathrm{CME}$ events. Hence we find in this map possible evidence of recurrent $\mathrm{CME}$ events embedded in the streamers; such events are poorly understood. Their analysis will be the matter of a future paper, as here we concentrate on the evolution of smaller-scale variability.

[20] We conclude that we can image the solar wind flows emanating from the streamer belt due to a combination of two factors: (1) the great sensitivity of the HI cameras to electron density variations of the K-corona and (2) the variability of the slow solar wind flow at the coronal heights imaged by $\mathrm{HI}-1 \mathrm{~A}$ is effectively recorded in difference images. The novel imaging technique presented here allows a qualitative analysis of the occurrence of transient plasma releases inside the streamer belt. We find that the variability of the slow solar wind in HI images manifests itself on a whole range of scales from the fine structure that is difficult to resolve into independent entities $\left(1-2^{\circ}\right.$ width), "distinct blobs" observed previously by SOHO coronagraphs $\left(3-20^{\circ}\right)$ and listed as "very poor events" in the CME LASCO catalogue, to larger events $\left(>20^{\circ}\right)$ which have the appearance of flux ropes (CMEs) and are listed as "poor events." The "very poor events" or "blobs" are found to be continually released from all parts of the streamer including latitudes which do not undergo dramatic latitudinal excursions. Our analysis suggests that transient releases (fine structure and blobs) can occur at high latitudes inside relatively flat parts of the streamers. These transients could result from open field lines of the rigidly rotating coronal holes reconnect with the closed field lines of the streamers [Sheeley et al., 1987; Wang et al., 1988]. Recent MHD models of the corona confirm the continual release of transients near the edges of the streamer belt [Lionello et al., 2005]. However, these blobs could also be related to the emergence of flux ropes poorly resolved in coronagraph images because they do not force significant electron density variations in the corona. Examples of such small events were discussed in a case study by Rouillard et al. [2009a]. 


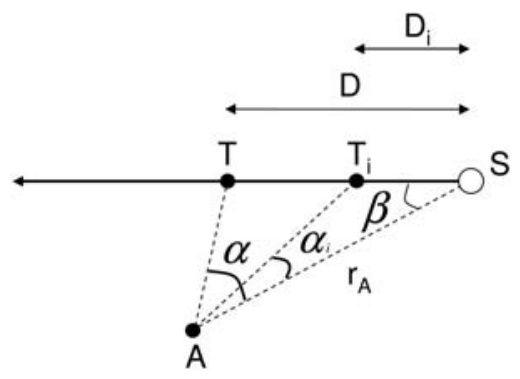

Figure 6. The geometry of the J-map fitting for an Imager A at a distance $r_{A}$ away from the Sun (S). A transient T propagates at an assumed constant speed $\left(V_{r}\right)$ along a solar radial with longitudinal separation angle $\beta$ relative to the Sun-Imager line.

[21] Some blobs can be sufficiently large to be followed through the entire HI-1 fov and well inside the TS [Rouillard et al., 2008]. Section 4 analyzes their kinematic properties as they get swept up by solar wind streams. We improve on methods (already partly published) to determine their trajectory. The latter will prove invaluable in the detailed in situ analysis of their properties in paper 2 .

\section{Radial Propagation of Transient Releases}

[22] Figure 5a presents a time-elongation map derived by plotting strips from HI-1A running difference images extracted along the varying Position Angle (PA) of the Earth (and therefore the Advanced Composition Explorer, ACE spacecraft) of the HI-A fields of view as a function of elongation (along the ordinate) and time (along the abscissa). An example of a strip of a HI-1A running difference image, centered along the PA of the Earth is shown in Figure 3. The J-map shown in Figure 5a covers the interval from 12 August to 17 September 2007 and is based on the technique reported by Sheeley et al. [1999, 2008a, 2008b] and Davies et al. [2009]. These plots, traditionally called J-maps were first created from coronal images taken by the LASCO C2/C3 coronagraphs on board SOHO [Sheeley et al., 1999]. The technique was recently adapted to HI images and perfected by Davies et al. [2009] and Sheeley et al. [2008a, 2008b]. The outflowing transients in Figure 5a can be divided into two categories: those associated with density perturbations that extend out to less than $10^{\circ}$ elongation and those (the larger perturbations) that propagate higher up in the corona, as far as $\alpha=60^{\circ}$. Figure 5a reveals five significant families of tracks, labeled A to E. Rouillard et al. [2008] investigated the possible source of the plasma parcels corresponding to tracks $\mathrm{D}$ to $\mathrm{E}$ and found no clear $\mathrm{CME}$ association. The transients first expanded into "pancakeshaped" structures, consistent with the evolution of the radial expansion of a plasma parcel, and progressively broadened and intensified in brightness in the HI-2 fov eventually forming a large-scale "plasma density wave." These tracks were associated with transient entrained by high-speed streams. Figure $4 \mathrm{a}$ is reproduced as Figure $5 \mathrm{~b}$ to allow comparisons; Figure 5c presents the solar wind speed predictions from the PFSS-ENLIL modeling which correspond to the plasma density simulations presented in Figure $4 \mathrm{~b}$. The input values of the density and speed are prescribed as inversely related parameters in the numerical model and therefore Figure $4 \mathrm{~b}$ and Figure $5 \mathrm{c}$ are directly related. White areas correspond to fast wind $\left(\sim 500 \mathrm{~km} \mathrm{~s}^{-1}\right)$ while the slow wind appears in black $\left(\sim 300 \mathrm{~km} \mathrm{~s}^{-1}\right)$. We have marked in Figure $5 \mathrm{c}$ alternating fast ( $\mathrm{F}$ : white/light grey regions) and slow flows (S: dark grey/black regions) in the vicinity of the solar ecliptic plane. Hence, the vertical dashed lines crossing Figures $5 \mathrm{~b}$ and $5 \mathrm{c}$ mark the times (solar longitudes) when fast wind is emitted after (eastward of) slow solar wind. These lines indicate times subsequent to which CIRs would be expected to form and, thus, large-scale plasma density waves should propagate in the HI-2 fov. The times of expected CIR formation correlate well with the occurrence of radially outflowing density variations (Figure 5a, features $\mathrm{A}$ to E).

[23] The periods around 9 and 17 September 2007 mark the times of latitudinal excursions of the streamer belt where fast flows are directly eastward of slow flows. Solar rotation will bring these two flow regimes in radial alignment, with slow flow ahead of fast flow, and force the formation of larger structures in the HI-2 fov. The imprints of these latter structures in the J-maps are tracks that extend far in the heliosphere as more and more plasma is piled up ahead of the driving fast wind. Tracks that do not extend beyond $10^{\circ}$ elongation emerge nearly continually in the ecliptic portion of the HI fov; this is related to the fact that there are transients continually released in the slow wind and passing along the line of sight in the vicinity of the ecliptic for much of the time. We suggest that these transients disappear rapidly in the $\mathrm{HI}$ field of view because they are not entrained by high-speed streams. A period of "rest" in the variable outflow occurs only between 27 August and 1 September 2007: Figure 5c reveals that the in-ecliptic portion of the HI fov is occupied by uniform fast solar wind at this time. This is related to the streamer belt being located at very high latitude during this period (see Figures $5 \mathrm{~b}$ and $5 \mathrm{c}$ ). The general topology of the streamer belt during the interval analyzed consists of one large excursion and two smaller ones per solar rotation, resulting in three ecliptic crossings of the streamer belt per solar rotation or an untypical threestream structure. Through an analysis of the last three years of solar wind in situ data, we have found that a three-stream structures has been a common feature over recent years. This observation agrees with the recent work of Temmer et al. [2007], who analyzed the variability of coronal hole areas in 2005 and found a 9 day periodicity. The authors found, from GOES/SXI image sequences, a periodic variation caused by a mutual triangular distribution of coronal holes $120^{\circ}$ apart in longitude.

\section{Outward Propagation of the Transients}

[24] An observer, such as the HI instrument on STEREOA, located at a constant radial distance from the Sun, $\mathrm{r}_{A}$, and measuring the apparent angular distance of an object moving at constant radial speed antisunward will observe an apparent acceleration of that object [Sheeley et al. 2008a, 2008b; Rouillard et al., 2008]. The elongation variation $(\alpha(t))$ of a density front moving radially outward with a 

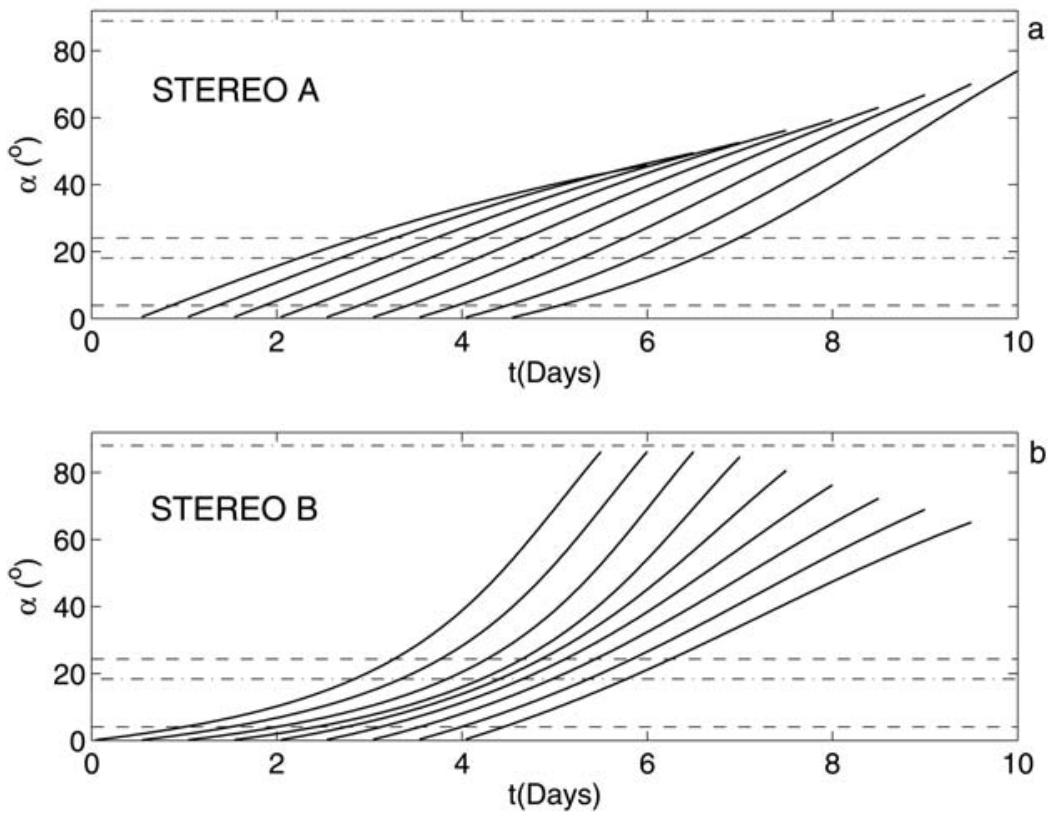

Figure 7. The expected elongation variation of corotating density plasma parcels in J-maps as seen by HI on (a) STEREO-A and (b) STEREO-B. The extents of the HI fovs are indicated by horizontal dotted lines for HI-1 and by dot-segment lines for HI-2.

constant speed $\left(V_{r}\right)$ along a solar radial with a longitude difference $(\beta)$ relative to the observer will be given by

$$
\alpha\left(V_{r}, \beta\right)=a \tan \left[\frac{D(t) \sin (\beta)}{r_{A}-D(t) \cos (\beta)}\right]
$$

where $D$ is the distance away from the Sun. An equivalent equation holds from elongation variations observed by STEREO-B with $r_{A}$ simply replaced by the radial distance of STEREO-B away from the Sun $r_{B}$ (typically $>1$ AU). A schematic of the geometry considered here is given in Figure 6.
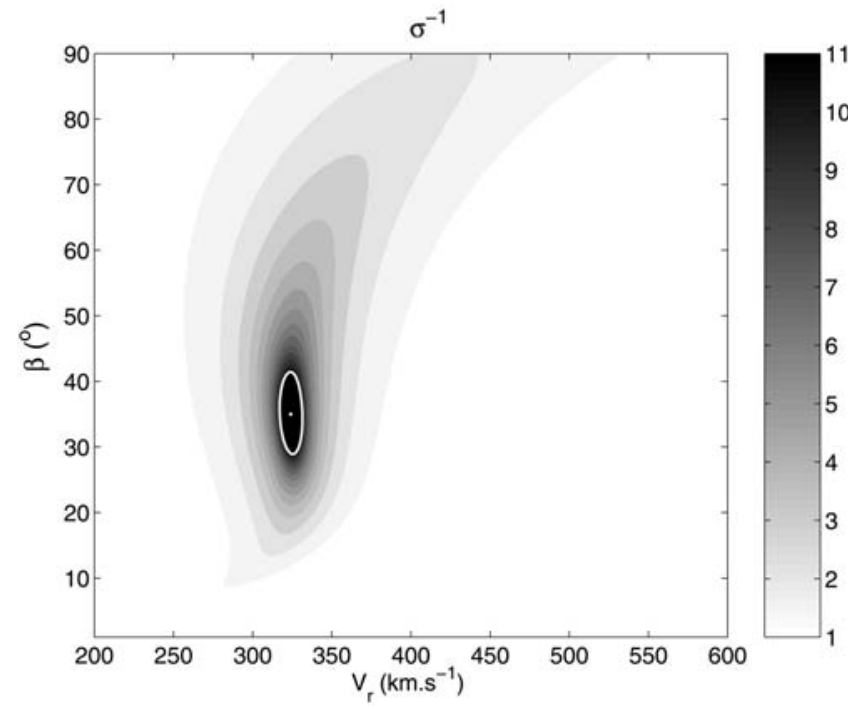

Figure 8. The inverse of the standard deviation of the residuals, contoured as a function of the radial speed $\left(V_{r}\right)$ and angle $\beta$.
[25] A similar equation was obtained by Sheeley et al. [2008a, 2008b] in terms of the angle out of the plane of the sky $\rho=90-\beta$. For an elongation at $t_{i}\left(\alpha_{i}=\alpha\left(t_{i}\right)\right)$, we can also derive the corresponding radial distance of the plasma parcel in solar radii $\left(r_{\odot}\right)$ :

$$
D_{i}=\left(r_{A}\left(t_{i}\right) / r_{\odot}\right)\left[\frac{\tan \left(\alpha_{i}\right)}{\cos (\beta) \tan \left(\alpha_{i}\right)+\sin (\beta)}\right]
$$

[26] We have shown in section 4 that the J-maps from $\mathrm{HI}$ on STEREO-A reveal a series of plasma parcels during

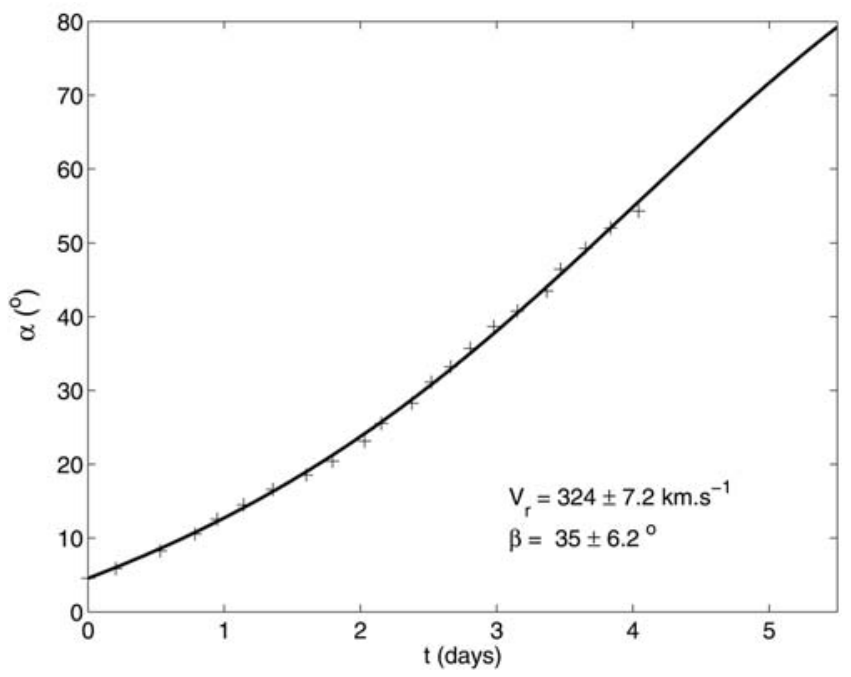

Figure 9. The elongation variation extracted from the J-map for a CME track (crosses) as a function of time since first appearance in the HI camera. The best fit curve is shown as a solid line. 

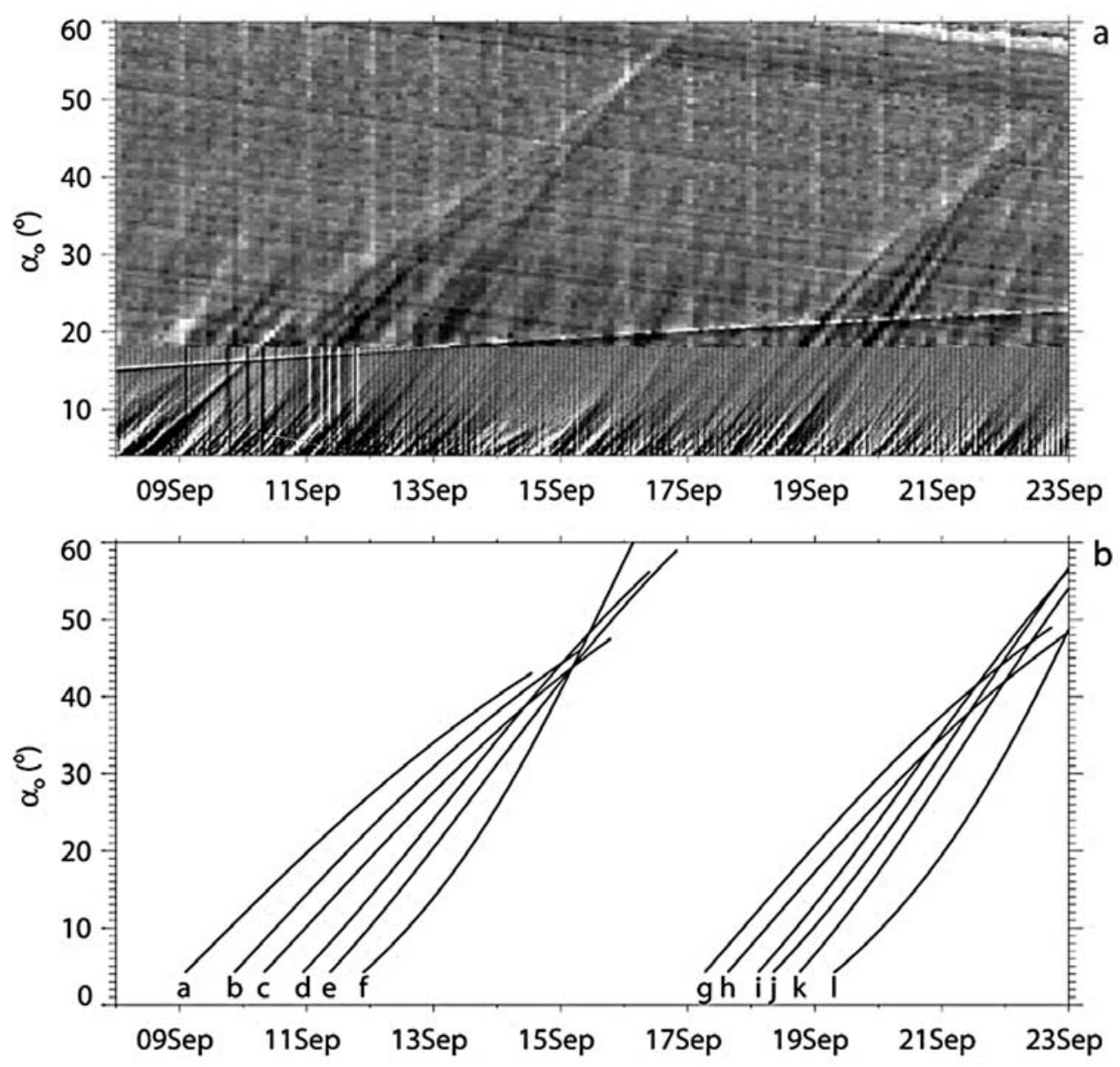

Figure 10. (a) The J-maps created from difference images created from the imagers on the STEREO-A spacecraft. (b) Each of the tracks shown in Figure 10a were fitted using the technique described in section 6. The families of tracks a to $\mathrm{f}$ and $\mathrm{g}$ to 1 correspond to the passage of CIR-D and CIR-E in Figure 5a, respectively.

the period of an expected CIR passage through the HI fov. The predicted elongation variation of a series of plasma density parcels emitted at a typical slow solar wind speed of $330 \mathrm{~km} \mathrm{~s}^{-1}$ at successive $12 \mathrm{~h}$ intervals from the same source region on the Sun is shown in Figure 7. The pattern of apparently converging tracks observed the HI-A J-map in Figure 5 is very similar to that shown in Figure $7 \mathrm{a}$. Therefore this convergence does not reveal the physical collision of plasma parcels, but is associated instead with distinct parcels being emitted by the same rotating source region (the streamer belt warp). The diverging pattern of tracks observed by HI on STEREO-B (Figure 7b) arises as corotation will rotate the source region away from the observer.

\section{CIR Structure Studied Using J-Maps}

[27] It is possible to fit the elongation variation $\left(\alpha\left(V_{r}, \beta\right)\right)$ for each feature in the J-maps and extract, for that feature, its particular speed and longitude of propagation. It is desirable to estimate the errors in the fitting procedure. $\alpha$ is evaluated for all possible values of $V_{r}$ and $\beta$. The standard deviation of the residuals is obtained by taking the differ-
Table 1. Trajectories of the Transients Determined From HI-A Observations $^{\mathrm{a}}$

\begin{tabular}{|c|c|c|c|c|}
\hline Track & Date & Time (UT) & $V_{r}\left(\mathrm{~km} \mathrm{~s}^{-1}\right)$ & $\beta(\operatorname{deg})$ \\
\hline \multicolumn{5}{|c|}{$C I R-D$} \\
\hline $\mathrm{a}$ & 9 Sep & 0107 & $268 \pm 14$ & $84 \pm 4$ \\
\hline $\mathrm{b}$ & 9 Sep & 1945 & $288 \pm 24$ & $82 \pm 9$ \\
\hline $\mathrm{c}$ & $10 \mathrm{Sep}$ & 0657 & $285 \pm 16$ & $78 \pm 10$ \\
\hline d & 10 Sep & 2139 & $299 \pm 11$ & $63 \pm 11$ \\
\hline $\mathrm{e}$ & 11 Sep & 0752 & $311 \pm 18$ & $61 \pm 11$ \\
\hline $\mathrm{f}$ & 11 Sep & \multicolumn{3}{|l|}{$C I R-E$} \\
\hline $\mathrm{g}$ & 17 Sep & 0531 & $307 \pm 21$ & $80 \pm 8$ \\
\hline $\mathrm{h}$ & 17 Sep & 1412 & $306 \pm 34$ & $80 \pm 11$ \\
\hline $\mathrm{i}$ & $18 \mathrm{Sep}$ & 0138 & $321 \pm 07$ & $59 \pm 10$ \\
\hline $\mathrm{j}$ & 18 Sep & 0720 & $319 \pm 07$ & $52 \pm 09$ \\
\hline $\mathrm{k}$ & 18 Sep & 1719 & $340 \pm 09$ & $55 \pm 12$ \\
\hline 1 & 19 Sep & 0612 & $324 \pm 07$ & $40 \pm 06$ \\
\hline
\end{tabular}

${ }^{\mathrm{a}}$ The date of transient passed $5^{\circ}$ of elongation, the estimated speed of transient $\left(V_{r}\right)$, and the longitude separation between the transient and the Observer $(\beta)$ values. 

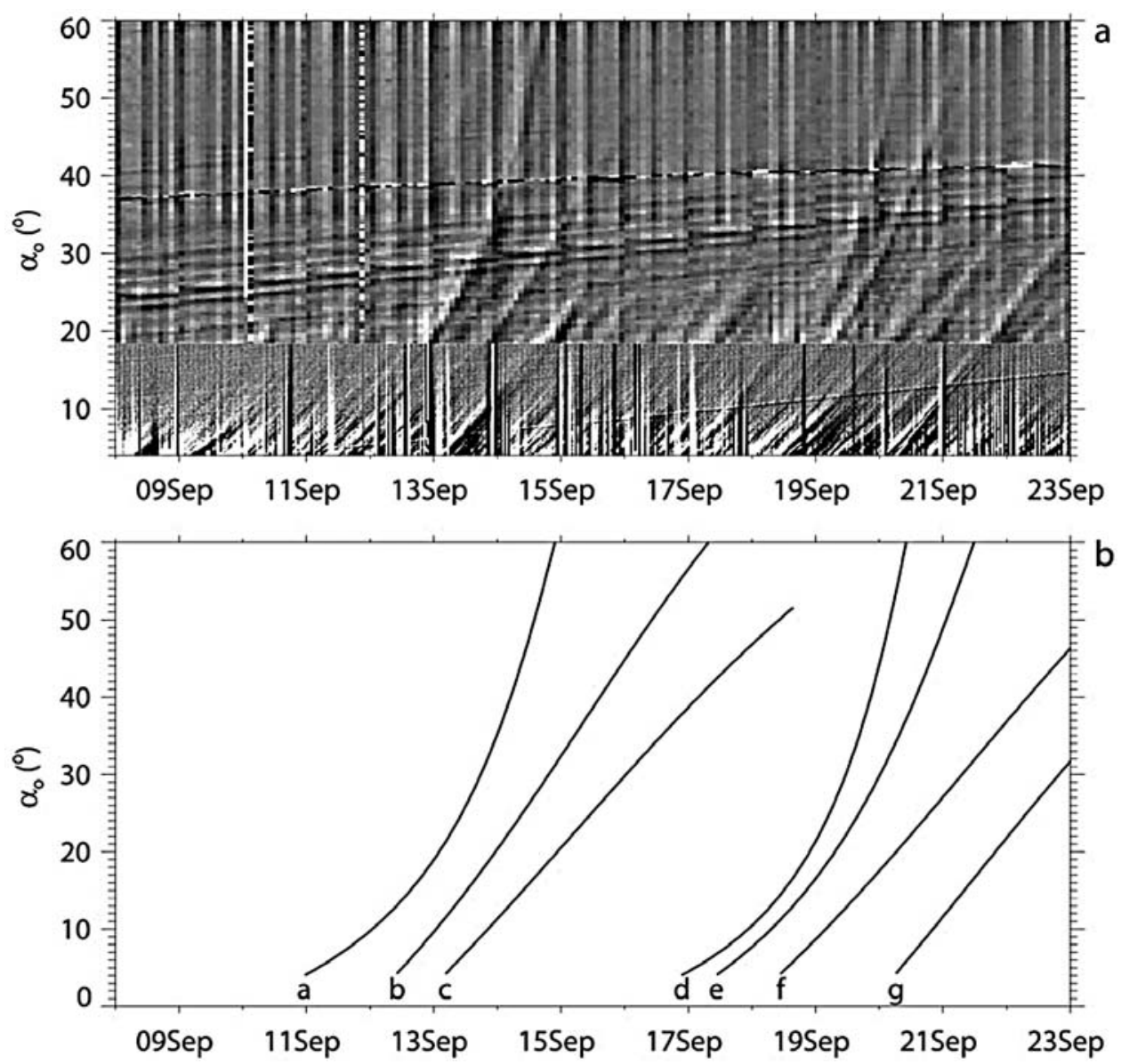

Figure 11. The same as Figure 10 but for STEREO B. In these J-maps the lines emanating from a single corotating source region diverge instead of converging. The families of tracks a to $\mathrm{c}$ and $\mathrm{d}$ to $\mathrm{g}$ correspond to the family of tracks associated with the passage of CIR-C and CIR-D in Figure 5a, respectively.

ence between the observed elongation variation $\alpha_{O}$ and the theoretical variation $\alpha$ is

$$
\sigma=\left[\sum\left(\alpha-\alpha_{O}\right)^{2}\right]^{1 / 2} / N
$$

[28] The standard deviation, $\sigma$, is calculated for all possible $V_{r}$ and $\beta$. Figure 8 presents the inverse of $\sigma$ as a contour plot for each value of $V_{r}$ (abscissa) and $\beta$ (ordinate). The best-fit for this CME track, observed in HI on STEREO-A during July 2007, was found to be $V_{r}=324 \mathrm{~km} \mathrm{~s}^{-1}$ and $\beta=35^{\circ}$. This CME track illustrates nicely both the effect of apparent acceleration and deceleration.

[29] The $2 \sigma$ contour line, a white line that encircles the maximum value (best fit), is used to evaluate the errors in the best fit $V_{r}$ and $\beta$ values. The errors are taken as half the range of $V_{r}$ and $\beta$ values covered by the $2 \sigma$ surface around the best fit combination of $\beta$ and $V_{r}$. The errors in the fitting of this particular feature, evaluated at the $2 \sigma$ level, were found to be $\pm 7.2 \mathrm{~km} \mathrm{~s}^{-1}$ and $\pm 6.2^{\circ}$ for the speed estimate and longitude separation, respectively. Figure 9 shows the fitted elongation versus time profile corresponding to the two-parameter fit shown in Figure 8. The same procedure was applied to all the plasma parcels observed between
8 and 23 September 2007, and which occur during the warps of the streamer belt imaged in Figure 4a.

[30] Figure 10a shows the J-map created for a subset of the interval shown in Figure 5a. The best fit elongation variations for each observed transient using the above technique are shown in Figure 10b. Table 1 shows the derived $V_{r}$ and $\beta$ together with the estimated uncertainties in the fitting of each of the tracks shown in Figure 10b. The $\beta$ values have a distinct pattern in that the longitude of propagation decrease systematically for consecutive parcels. The

Table 2. Same as Table 1 but for the Tracks Fitted in HI-B Images

\begin{tabular}{ccccc}
\hline Track & Date & Time $(\mathrm{UT})$ & $V_{r}\left(\mathrm{~km} \mathrm{~s}^{-1}\right)$ & $\beta(\mathrm{deg})$ \\
\hline & & $C I R-C$ & & \\
a & 10 Sep & 2233 & $330 \pm 23$ & $17 \pm 4$ \\
b & 12 Sep & 0909 & $326 \pm 06$ & $53 \pm 9$ \\
c & 13 Sep & 0330 & $282 \pm 12$ & $68 \pm 10$ \\
& & $C I R-D$ & & \\
d & 16 Sep & 2031 & $357 \pm 190$ & $14 \pm 15$ \\
e & 17 Sep & 0952 & $333 \pm 15$ & $36 \pm 09$ \\
f & 18 Sep & 0943 & $274 \pm 05$ & $59 \pm 10$ \\
g & 20 Sep & 0520 & $319 \pm 10$ & $85 \pm 07$ \\
\hline
\end{tabular}




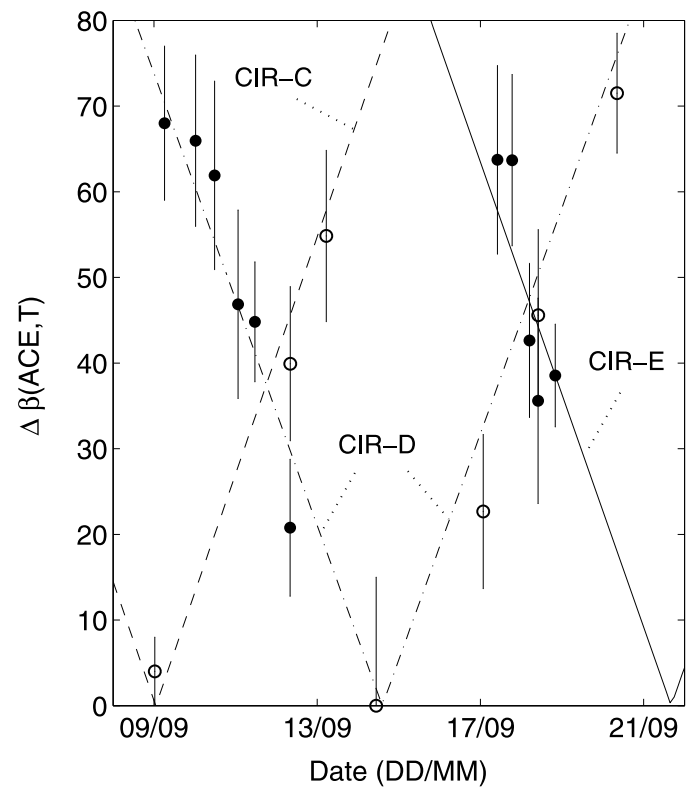

Figure 12. The longitude difference between ACE and the fitted propagating front as a function of time. The $\Delta \beta$ (ACE, $\mathrm{T})$ variation of a CIR source region rotating with a period of 27 days is shown by the three lines. These $\mathrm{V}$-shaped lines track well the observed variation in $\Delta \beta$ (ACE, T) during these intervals.

$\beta$ values associated with the second family of parcels behave in the same manner. The progressive decrease in $\beta$ values is also marked by a slight increase in $V_{r}$ during fits $a$ to $f$ (except between $b$ and $c$ ). The fits to the second family of tracks for $g$ to $l$ show a much less pronounced $V_{r}$ variation and yet the $\beta$ values also decreased substantially $(\beta=80 \pm$ $8^{\circ}$ to $\beta=40 \pm 6^{\circ}$ ). The slight decrease in solar wind speed is not expected for time-independent coronal hole flows at the source surface and may suggest that the assumption of constant speed is not totally adequate. The simple twoparameter fit used here has however the advantages of being intuitive and demonstrating clearly the corotation of the source region.

[31] The fitting technique was applied to the tracks observed during the same period by HI on STEREO-B. The $\mathrm{J}$-maps and the best fit lines for the tracked transients are shown in Figures $11 \mathrm{a}$ and $11 \mathrm{~b}$, respectively. The values obtained are shown in Table 2. It is clear that for the two families of diverging tracks, the $\beta$ increases for consecutive tracks, as would be expected if the source region was rotating away from the instrument. The $V_{r}$ values also decrease as $\beta$ increases, again pointing to the limitations of the technique. Assuming constant speed of plasma parcels is not fully adequate as parcels may still be accelerating in the inner HI-1A field of view and a fitting technique which convolves the geometrical effect analyzed here with a gradual acceleration of the parcel (multiparameter fit) could be attempted in future studies. Despite limitations in the technique used here, this analysis shows that the signatures seen in STEREO-A and STEREO-B are consistent with the tracks of outflowing parcels emitted from a rotating source region. Moreover, as we shall see in paper 2 , this simple technique provides impressive kinematic predictions when compared with in situ data.

[32] The analysis can be taken one step further to assess whether the variation in the estimated longitudinal separation angle between the imager and the source region is related to the solar rotation period. Figure 12 shows using black filled and white empty circles the angular separation between the Earth's heliocentric longitude (and therefore ACE) and the longitude of the source region of transient T, for each fitted track $(\Delta \beta(\mathrm{ACE}, \mathrm{T}))$ as a function of time, the time axis covers the interval considered in Figure 10. The time of each circle is the time that the transient passed $0.1 \mathrm{AU}$, calculated by using equation 2 and the estimated speed of each transient. Filled black circles are derived from tracks observed by HI on STEREO-A while the empty circles are for STEREO-B. The dash and dash-dot-dash lines mark the expected variation in the longitudinal separation angle between the Earth and the transients source region for the rotation period of $\sim 27$ days observed at the time. We can see that, within their estimated errors, the observations mainly follow the expected values. The interaction of transients are imaged for three CIRs during this time interval which we label CIR-C, CIR-D and CIR-E in Figure 12. As expected, during the time interval of Figure 12 (half a solar rotation) only one corotating source of transients, labeled CIR-D, is tracked continuously in both STEREO-A and STEREO-B images (i.e., filled and empty circles occur on the same V-shaped line).

[33] We have established that, during the time interval studied in this paper, $\mathrm{HI}$ is observing plasma parcels emitted by specific rotating sources. The presence of these parcels close-in led Sheeley et al. [2008a, 2008b] and Rouillard et al. [2008] to suggest that they were transients (flux ropes or reconnected magnetic field lines). The scale of these perturbations in HI-2 cameras and the systematic correlation between their predicted arrival time at Earth and typical in situ signatures of CIRs (see Rouillard et al. [2008] and paper 2) leads to the conclusion that these elements are entrained by CIRs. It is important to remember that the analysis so far has been carried out using difference images rather than background subtracted images. As stated earlier, faint features can be tracked out more easily in difference images. The passage of corotating streams through the fov of HI-1 should appear in undifferenced images as a more continuous enhancement of the solar wind brightness over 7 days $\left(90^{\circ}\right.$ of corotation in longitude angle to enter and leave the portion of the TS imaged by HI-1). This enhancement would vary with the proximity of the (CIR associated) spiral orientation to the line of sight (see paper 2 for a discussion of this effect) and the distance between the electrons of the spiral and the TS. The slow manner in which the corotating Archemedian spiral of denser slow solar wind passes through the fov means that it is mostly suppressed in difference images. The brightness variations that remain in difference images are therefore the small-scale transients (density ripples) added to the brightness of the corotating stream. In HI-2 where the fast solar wind is expected to catch up the slow solar wind, previous studies showed that largescale "plasma density waves" form which dominate the 
brightness variations in a transient manner [Sheeley et al., 2008a, 2008b].

\section{Discussion and Conclusion}

[34] In this paper, we have demonstrated how HI provides a unique way to study the variability of the slow solar wind. The sensitivity of the HI-1A cameras allows imaging of helmet streamers as far as $D \sim 54 R_{\odot}$ and in great detail. The first obvious observation is the continual emergence of transients which appear clearly in latitude versus longitude maps obtained from difference images. The perturbations appear as intermittent temporary increases in coronal brightness in the streamers or/and sudden temporary widening of these streamers. These transients appear to have a whole range of sizes and can occasionally be extensive structures; the study presented here even finds evidence for the recurrent eruption of twisted magnetic structures off the northeastern limb of the Sun. This recurrent event has the appearance of a small CME in coronagraph and HI images, however more work is required to confirm this. A quantitative analysis of the trajectory of the ecliptic part of these small-scale transients shows that their source region rotates with the solar rotation period. Their source region was found to be located on the western boundary of coronal holes where fast wind is emitted ahead of slow wind.

[35] The median of the time difference between successive transients tracked all the way into HI-2 is found to be $\sim 12 \mathrm{~h}$ during the time interval considered in this paper (estimated from Tables 1 and 2). The plasma releases force local variations in density that propagate outward in the slow solar wind. As we shall see in paper 2, in situ analysis of the slow solar wind observed by STEREO-A/B and ACE during the period observed by HI shows clear evidence of this variability of the slow solar wind. All the types of transients typically seen in the slow solar wind were observed during this short interval.

[36] The results of this paper which consist of a systematic estimation of transient trajectories and a rough estimation of their sizes are used in the accompanying paper (paper 2) to carry out a detailed analysis of the in situ signatures of these transients.

[37] Acknowledgments. We gratefully acknowledge fruitful discussions with Yi-Ming Wang of NRL. We thank the ACE SWEPAM team for providing the solar wind in situ data. This work was funded by the Science and Technology Facilities Council (UK). The STEREO/SECCHI data are produced by a consortium of RAL (UK), NRL (USA), LMSAL (USA), GSFC (USA), MPS (Germany), CSL (Belgium), IOTA (France), and IAS (France). The SECCHI data presented in this paper were obtained from the UKSSDC World Data Center, Chilton, UK.

[38] Zuyin Pu thanks the reviewers for their assistance in evaluating this paper.

\section{References}

Arge, C. N., and V. J. Pizzo (2000), Improvement in the prediction of solar wind conditions using near-real time solar magnetic field updates, J. Geophys. Res., 105(A5), 10,465.

Balogh, A., and E. J. Smith (2001), The heliospheric magnetic field at solar maximum: Ulysses observations, Space Sci. Rev., 97(1/2), 147.

Breen, A. R., P. Riley, A. J. Lazarus, A. Canals, R. A. Fallows, J. Linker, and Z. Mikic (2002), The solar wind at solar maximum: Comparisons of EISCAT IPS and in-situ observations, Ann. Geophys., 20(9), 1291.

Brueckner, G. E., et al. (1995), The Large Angle Spectroscopic Coronagraph (LASCO), Sol. Phys., 162, 357.
Brown, D. S., D. Bewsher, and C. J. Eyles (2009), Calibrating the pointing and optical parameters of the STEREO heliospheric imagers, Sol. Phys. 254(1), 185

Dahlburg, R. B., and J. T. Karpen (1997), Reconnection in adjoining coronal helmet streamers, Adv. Space Res., 19, 1887.

Davies, J. A., R. A. Harrison, A. P. Rouillard, N. R. Sheeley Jr., C. H. Perry, D. Bewsher, C. J. Davis, C. J. Eyles, S. R. Crothers, and D. S. Brown (2009), A synoptic view of solar transient evolution in the inner heliosphere using the Heliospheric Imagers on STEREO, Geophys. Res. Lett., 36, L02102, doi:10.1029/2008GL036182.

Eyles, C. J., et al. (2009), The Heliospheric Imagers onboard the STEREO mission, Sol. Phys., 254(2), 387.

Grall, R. R., W. A. Coles, M. T. Klinglesmith, A. R. Breen, P. J. S. Williams, J. Markkanen, and R. Esser (1996), Rapid acceleration of the polar solar wind, Nature, 379, 429.

Harrison, R. A., et al. (2008), First imaging of coronal mass ejections in the heliosphere viewed from outside the Sun Earth line, Sol. Phys., 247(1), 171.

Howard, R. A., et al. (2008), Sun Earth Connection Coronal and Heliospheric Investigation (SECCHI), Space Sci. Rev., 136(1-4), 67.

Jackson, B. V. (1991), HELIOS spacecraft photometer observation of elongated corotating structures in the interplanetary medium, J. Geophys. Res., 96, 11,307.

Kaiser, M. L., T. A. Kucera, J. M. Davila, O. C. St. Cyr, M. Guhathakurta and E. Christian (2008), The STEREO mission: An overview, Space Sci. Rev., 136(1-4), 5 .

Koutchmy, S. (1992), Streamer eclipse observations, in Proceedings of the First SOHO Workshop, Eur. Space Agency Spec. Publ., ESA SP-348, 73. Koutchmy, S., and G. Stellmacher (1976), Photometric study of chromospheric and coronal spikes observed during the total solar eclipse of 30 June, 1973, Sol. Phys., 49, 253.

Lee, M. A. (2000), An analytical theory of the morphology, flows, and shock compressions at corotating interaction regions in the solar wind, J. Geophys. Res., 105(A5), 10,491.

Lionello, R., P. Riley, J. A. Linker, and Z. Mikic (2005), The effects of differential rotation on the magnetic structure of the solar corona: Magnetohydrodynamic simulations, Astrophys. J., 625(1), 463.

Luhmann, J. G., S. C. Solomon, J. A. Linker, J. G Lyon, Z. Mikic, D. Odstrcil, W. Wang, and M. Wiltberger (2004), Coupled model simulation of a Sun-to-Earth space weather event, J. Atmos. Sol. Terr. Phys. 66(15-16), 1243

McComas, D. J., et al. (1998), Ulysses' return to the slow solar wind, Geophys. Res. Lett., 25(1), 1.

McComas, D. J., R. Goldstein, J. T. Gosling and R. M. Skoug (2001), Ulysses' second orbit: Remarkably different solar wind, Space Sci. Rev., 97(1/4), 99.

Mikić, Z., and J. A. Linker (1996), The large-scale structure of the solar corona and inner heliosphere, AIP Conf. Proc., 382, 104.

Mikić, Z., J. A. Linker, P. Riley, and R. Linello (2000), Predicting the structure of the solar corona during the 11 August 1999 total solar eclipse, in The Last Total Solar Eclipse of the Millennium in Turkey, ASP Conf. Ser., vol. 205, edited by W. Livingston and A. Özgüc, p. 162, Astron. Soc. of the Pac., San Francisco, Calif.

Odstrčil, D. and V. J. Pizzo (1999), Three-dimensional propagation of coronal mass ejections in a structured solar wind flow: 2. CME launched adjacent to the streamer belt, J. Geophys. Res., 104(A1), 493.

Parker, E. N. (1958), Dynamics of the interplanetary gas and magnetic fields, Astrophys. J., 128, 664

Pizzo, V. J., and J. T. Gosling (1994), 3-D simulation of high-latitude interaction regions: Comparison with ULYSSES results, Geophys. Res. Lett., 21(18), 2063

Pneuman, G., and R. A. Kopp (1971), Gas-magnetic field interactions in the solar corona, Sol. Phys., 18, 258.

Poland, A. I. (1978), Motions and mass changes of a persistent coronal streamer, Sol. Phys., 57, 141 .

Rouillard, A. P., and M. Lockwood (2007), The latitudinal effect of corotating interaction regions on galactic cosmic rays, Sol. Phys., 245(1), 191.

Rouillard, A. P., et al. (2008), First imaging of corotating interaction regions using the STEREO spacecraft, Geophys. Res. Lett., 35, L10110, doi:10.1029/2008GL033767.

Rouillard, A. P., et al. (2009a), A multispacecraft analysis of a small-scale transient entrained by solar wind streams, Sol. Phys., 256(1-2), 307.

Rouillard, A. P., et al. (2009b), A solar storm observed from the Sun to Venus using the STEREO, Venus Express, and MESSENGER spacecraft, J. Geophys. Res., 114, A07106, doi:10.1029/2008JA014034.

Saez, F., A. Llebaria, P. Lamy, and D. Vibert (2000), Three-dimensional reconstruction of the streamer belt and other large-scale structures of the solar corona. I. Method, Astron. Astrophys., 473(1), 265. 
Schmahl, E. J., N. Gopalswamy, and M. R. Kundu (1992), Models of coronal streamers at meter-decameter wavelengths, in Proceedings of the First SOHO Workshop, Eur. Space Agency Spec. Publ., ESA SP-348, 145.

Sheeley, N. R., Jr. (2008), A century of polar faculae variations, Astrophys. $J ., 680(2), 1553$.

Sheeley, N. R., Jr., A. G. Nash, and Y. M. Wang (1987), The origin of rigidly rotating magnetic field patterns on the Sun, Astrophys. J., 319, 481

Sheeley, N. R., Jr., et al. (1997), Measurements of flow speeds in the corona between 2 and $30 R_{\odot}$, Astrophys. J., 484, 472.

Sheeley, N. R., J. H. Walters, Y.-M. Wang, and R. A. Howard (1999), Continuous tracking of coronal outflows: Two kinds of coronal mass ejections, J. Geophys. Res., 104(A11), 24,739.

Sheeley, N. R., Jr., T. N. Knudson, and Y. M. Wang (2001), Coronal inflows and the Sun's nonaxisymmetric open flux, Astrophys. J., 546(2), L131.

Sheeley, N. R., Jr., et al. (2008a), Heliospheric images of the solar wind at Earth, Astrophys. J., 675(1), 853.

Sheeley, N. R., Jr., et al. (2008b), SECCHI observations of the Sun's gardenhose density spiral, Astrophys. J., 674(2), L109.

Suess, S. T., A. Bemporad, and G. Poletto (2004), A slow streamer blowout at the Sun and Ulysses, Geophys. Res. Lett., 31, L05801, doi:10.1029/ 2003GL018895.

Temmer, T., B. Vršnak, and V. Astrid (2007), Periodic appearance of coronal holes and the related variation of solar wind parameters, Sol. Phys., 241(2), 371

Thernisien, A. F., and R. A. Howard (2006), Electron density modeling of a streamer using LASCO data of 2004 January and February, Astrophys. J., 642(1), 523

van Aalst, M. K., P. C. Martens, and A. J. C. Beliën (1999), Can streamer blobs prevent the buildup of the interplanetary magnetic field?, Astrophys. $J ., 511(2), \mathrm{L} 125$

Vourlidas, A., and R. A. Howard (2006), The proper treatment of coronal mass ejection brightness: A new methodology and implications for observations, Astrophys. J., 642(2), 1216.

Wang, Y.-M. (1994), Two types of slow solar wind, Astrophys. J., 437(1), L67.

Wang, Y.-M., and N. R. Sheeley Jr. (2003), On the topological evolution of the coronal magnetic field during the solar cycle, Astrophys. J., 599(2), 1404.

Wang, Y.-M., N. R. Sheeley Jr., A. G. Nash, and L. R. Shampine (1988), The quasi-rigid rotation of coronal magnetic fields, Astrophys. J., 327 , 427.
Wang, Y.-M., N. R. Sheeley Jr., and A. G. Nash (1990), Latitudinal distribution of solar-wind speed from magnetic observations of the Sun, Nature, 347,439 .

Wang, Y.-M., et al. (1997), The green line corona and its relation to the photospheric magnetic field, Astrophys. J., 485, 419.

Wang, Y.-M., N. R. Sheeley Jr., J. H. Walters, G. E. Brueckner, R. A Howard, D. J. Michels, P. L. Lamy, R. Schwenn, and G. M. Simnett (1998), Origin of streamer material in the outer corona, Astrophys. J., 498, L165.

Wang, Y.-M., N. R. Sheeley, D. G. Socker, R. A. Howard, and N. B Rich (2000), The dynamical nature of coronal streamers, J. Geophys. Res. 105(A11), 25,133.

Wang, Y.-M., N. R. Sheeley Jr., and A. P. Rouillard (2006), Role of the Sun's nonaxisymmetric open flux in cosmic-ray modulation, Astrophys. J., 644(1), 638.

Wang, Y.-M., J. B. Biersteker, N. R. Sheeley Jr., S. Koutchmy, J. Mouette, and M. Druckmller (2007), The solar eclipse of 2006 and the origin of raylike features in the white-light corona, Astrophys. J., 660(1), 882.

Yashiro, S., N. Gopalswamy, G. Michalek, O. C. St. Cyr, S. P. Plunkett, N. B. Rich, and R. A. Howard (2004), A catalog of white light coronal mass ejections observed by the $\mathrm{SOHO}$ spacecraft, J. Geophys. Res. 109, A07105, doi:10.1029/2003JA010282.

Zurbuchen, T. H., L. A. Fisk, G. Gloeckler, and R. von Steiger (2002), The solar wind composition throughout the solar cycle: A continuum of dynamic states, Geophys. Res. Lett., 29(9), 1352, doi:10.1029/2001GL013946.

D. Bewsher, S. R. Crothers, J. A. Davies, C. J. Davis, C. J. Eyles, and R. A. Harrison, Space Science and Technology Department, Rutherford Appleton Laboratory, Fermi Ave., Chilton OX11 0QX, UK

D. S. Brown, Institute of Mathematical and Physical Sciences, University of Wales, Penglais Hill, Aberystwyth SY23 3BZ, UK.

R. J. Forsyth and N. P. Savani, Space and Atmospheric Physics Group, Blackett Laboratory, Imperial College London, London SW7 2BZ, UK.

R. A. Howard, N. R. Sheeley, and A. Vourlidas, Space Science Division, Naval Research Laboratory, Washington, DC 20375-5352, USA.

B. Lavraud, Université de Toulouse, UPS, Centre d'Etude Spatiale des Rayonnements, 9 Ave. du Colonel Roche, Toulouse F-31028 CEDEX 4 France.

M. Lockwood and A. P. Rouillard, Space Environment Physics Group, School of Physics and Astronomy, University of Southampton, Southampton SO17 1BJ, UK. (alexisrouillard@yahoo.co.uk) 